\title{
Coincidence Detection or Temporal Integration? What the Neurons in Somatosensory Cortex Are Doing
}

\author{
Stephane A. Roy and Kevin D. Alloway \\ Department of Neuroscience and Anatomy, Penn State University College of Medicine, \\ Hershey, Pennsylvania 17033-2255
}

To assess the impact of thalamic synchronization on cortical responsiveness, we used conditional cross-correlation analysis to measure the probability of neuronal discharges in somatosensory cortex as a function of the time between discharges in pairs of simultaneously recorded neurons in the ventrobasal thalamus. Among 26 neuronal trios, we found that thalamocortical efficacy after synchronous thalamic activity was nearly twice as large as the efficacy rate obtained when pairs of thalamic neurons discharged asynchronously. Nearly half of these neuronal trios displayed cooperative effects in which the cortical discharge probability after synchronous thalamic

Neuronal synchronization is present in many brain regions during sensory stimulation, but its role in sensory processing is controversial (Gray, 1999; Shadlen and Movshon, 1999). Many investigators believe that neuronal synchronization is critical for transmitting sensory information and have suggested that a major function of cortical neurons is to detect coincident events among their presynaptic inputs (Abeles, 1982; Softky and Koch, 1993; Alonso et al., 1996; Konig et al., 1996). Although some evidence indicates that synchronous excitation has a strong influence on the timing of cortical discharges (Softky and Koch, 1993; Stevens and Zador, 1998; Harsch and Robinson, 2000; Salinas and Sejnowski, 2000), other evidence suggests that cortical discharge patterns reflect the time-dependent integration of excitatory and inhibitory inputs (Bernander et al., 1991; Reich et al., 1997; Troyer and Miller, 1997; Shadlen and Newsome, 1998). In fact, these latter results have prompted the view that mean firing rate must be more important for sensory coding than spike timing because information about the timing of synaptic inputs is not preserved by integrate-and-fire mechanisms (Shadlen and Newsome, 1994, 1998). These conclusions, however, are based almost entirely on studies that either used computer modeling techniques to simulate neuronal input-output relationships or studied neuronal behavior in response to direct electrical stimulation. Consequently, unless presynaptic inputs are directly monitored with respect to the discharge behavior of cortical neurons in vivo,

Received Sept. 13, 2000; revised Jan. 19, 2001; accepted Jan. 19, 2001.

This work was supported by grants awarded to K.D.A. from the National Institutes of Health (NINDS-29363, NINDS-37532) and the National Science Foundation (NSF-9983285), and by a Pennsylvania State University Computational Fellowship awarded to S.A.R. We thank Dr. Steven P. Dear for his helpful comments on an earlier draft of this manuscript.

Correspondence should be addressed to Dr. Kevin D. Alloway, Department of Neuroscience and Anatomy, H109, Milton S. Hershey Medical Center, 500 University Drive, Hershey, PA 17033-2255. E-mail: kda1@psu.edu.

Copyright (C) 2001 Society for Neuroscience $0270-6474 / 01 / 212462-12 \$ 15.00 / 0$ events was larger than could be predicted from the efficacy rate of individual thalamic discharges. In these cases of heterosynaptic cooperativity, thalamocortical efficacy declined to asymptotic levels when the interspike intervals were $>6-8 \mathrm{msec}$. These results indicate that thalamic synchronization has a significant impact on cortical responsiveness and suggest that neuronal synchronization may play a critical role in the transmission of sensory information from one brain region to another.

Key words: cross-correlation analysis; cutaneous stimulation; cooperativity; snowflake analysis; synchronization; thalamocortical much of the controversy surrounding neuronal synchronization and its impact on cortical activity is unlikely to be resolved.

We have shown previously that cutaneous stimulation evokes synchronized discharges among local groups of neurons in the ventrobasal thalamus (Alloway et al., 1995) but did not examine how this synchronization affects the discharge behavior of target neurons in somatosensory cortex. In the present study we tested the hypothesis that stimulus-induced synchronization in the ventrobasal thalamus facilitates the firing of target neurons in the secondary somatosensory (SII) cortex. As part of our analysis, we measured the discharge probability of individual cortical neurons as a function of differences in the relative timing of thalamic discharges so that we could determine the effective interval for integrating thalamic inputs. Similar work in the visual system has shown that synchronization in the lateral geniculate nucleus increases the activation of neurons in striate cortex (Alonso et al., 1996; Usrey et al., 2000). Our results in the somatosensory system are consistent with those in the visual system and compel us to suggest that neuronal synchronization is a fundamental mechanism for facilitating transmission of sensory information from one brain region to another.

\section{MATERIALS AND METHODS}

Animal preparation. All procedures followed guidelines established by the National Institutes of Health on the use of laboratory animals and were similar to those described in previous reports (Johnson and Alloway, 1994, 1996; Roy and Alloway, 1999). Briefly, experiments were conducted on two domestic cats in which a stainless steel recording chamber had been chronically implanted onto the cranium overlying the ectosylvian gyrus (SII cortex) and the ventrobasal thalamus during a sterile surgical operation. A bolt was also attached to the occipital ridge so that the head could be immobilized during neuronal recording. The animal was intubated through the oral cavity and was ventilated with a $2: 1$ gaseous mixture of nitrous oxide and oxygen containing $0.5 \%$ isoflurane to prevent reflexive movements. Because the animal's head was not held in a stereotaxic instrument, this concentration of isoflurane was lower than the concentration that was needed for inducing anesthesia when the soft tissue surrounding the ears, eyes, and mouth was contacted 
by ear bars and other stereotaxic devices. Body temperature was maintained at $37^{\circ} \mathrm{C}$, and both heart rate and end-tidal $\mathrm{CO}_{2}$ were monitored continuously.

The final experimental session was terminated by an intravenous injection of $30 \mathrm{mg}$ pentobarbital sodium. The animal was transcardially perfused with $500 \mathrm{ml}$ of $0.9 \%$ saline containing $20 \mathrm{mg}$ lidocaine and 1000 units of heparin, followed by $500 \mathrm{ml}$ of neutral formalin, and then $500 \mathrm{ml}$ of neutral formalin in $10 \%$ sucrose. The brain was removed and placed in fixative and $30 \%$ sucrose until it sank. To confirm that recordings were made in the ventrobasal thalamus and SII cortex, the brain was cut into $50 \mu \mathrm{m}$ coronal sections that were mounted onto chrom-alum-coated slides and stained with thionin.

Electrophysiology. Tungsten microelectrodes (2-4 $\mathrm{M} \Omega$ ) were used to record extracellular discharges simultaneously from neurons in the ventrobasal thalamus and SII cortex. Two electrodes, glued to each other so that their tips were separated by $400-600 \mu \mathrm{m}$, were advanced in tandem into the forelimb representation of the ventrobasal complex $(\sim 16-18 \mathrm{~mm}$ below dura) to record simultaneous discharges from different thalamic neurons. With this electrode separation, we often encountered pairs of neurons that had similar response properties, but the electrode separation was large enough to avoid recording the same thalamic neuron with both electrodes.

In cats, SII cortex receives direct projections from the ventrobasal complex (Spreafico et al., 1981; Burton and Kopf, 1984). We recorded neurons in SII cortex instead of primary somatosensory (SI) cortex because receptive fields in SII cortex are larger, and this increased the probability of encountering neurons with receptive fields overlapping those recorded in the thalamus (Alloway and Burton, 1985). In some experiments we placed only one electrode in SII cortex, but in others we used an array of two to four electrodes in which adjacent electrodes were separated by at least $250 \mu \mathrm{m}$. In both cases, we tried to place the electrodes in regions of SII cortex that matched the receptive field properties of neurons recorded in the ventrobasal complex. In both brain regions the electrodes were advanced until we encountered neurons with well defined receptive field boundaries that responded exclusively to cutaneous hair movements. In a laminar study of SII response properties in the anesthetized cat, $85 \%$ of cutaneous-sensitive neurons with well defined receptive fields were located in layers IIIb and IV (Alloway and Burton, 1985, their Table 6). This previous finding was consistent with the fact that most of our cortical electrode penetrations had recording depths indicating that the neurons were located in the middle cortical layers. We did not make any electrolytic lesions in the present study, however, and did not reconstruct the SII neuronal recording sites with respect to the cortical layers.

Cutaneous stimulation. We have shown previously that thalamic and cortical neurons that are sensitive to hair movements can be activated by computer-controlled airjets (Johnson and Alloway, 1994, 1996; Roy and Alloway, 1999). In the present study we used stationary airjets to stimulate sites within the receptive fields of simultaneously recorded neurons in the thalamus and SII cortex. While searching for neurons that responded to hair movements, we often used a fine brush or a hand-held airjet to stimulate the hairy skin. The boundaries of the receptive fields were determined by listening to an audio monitor of neuronal discharges in a single amplifier channel while using a hand-held airjet to stimulate the hairy skin. The receptive field boundaries were then traced onto outline drawings of the cat forearm.

Computer-controlled airjets were presented across a block of 200 trials. Each trial consisted of a prestimulation period ( $2 \mathrm{sec})$, a stimulation period consisting of a series of stationary airjets interspersed with spontaneous activity ( $5 \mathrm{sec})$, and a poststimulation period $(2 \mathrm{sec})$. During each stimulation period, two airjets were delivered individually and then in combination through a pair of $1 \mathrm{~mm}$ hollow tubes oriented orthogonal to the hairy skin. Although each airjet tube was only $1 \mathrm{~mm}$ in diameter, divergence of airflow from each tube usually caused hair movements within a circular region of skin that had a diameter of $\sim 5 \mathrm{~mm}$. Airflow was controlled by an electronic valve that was triggered by an output pulse from the data acquisition system (DataWave Technologies, Broomfield, CO). Air pressure was maintained at 20 psi by a needle valve in series with a pressure gauge. Each airjet lasted $1 \mathrm{sec}$ for a total of $3 \mathrm{sec}$ of stimulation in each trial. Airjets were separated by interstimulus intervals of $1 \mathrm{sec}$, and each trial was separated by intertrial intervals of 4-6 sec.

Cross-correlation analysis. Extracellular neuronal waveforms were amplified, displayed, and converted into digital signals that were timestamped to a resolution of $0.1 \mathrm{msec}$. Neuronal waveforms were sorted on the basis of several parameters, including spike width, amplitude, peak time, valley time, and other distinguishing features. Time stamps of sorted waveforms were stored on hard disk and then used to construct peristimulus timed histograms (PSTHs), cross-correlation histograms (CCGs), and snowflake histograms. Unless stated otherwise, all CCGs and snowflake histograms were constructed from the sum of all discharges occurring during the three airjet stimuli, but not from spontaneous discharges that appeared during the prestimulation period or during intervals between airjets.

To evaluate the strength of pairwise connections between simultaneously recorded thalamic and cortical neurons, raw CCGs were constructed to display changes in SII activity as a function of thalamic discharges occurring at time zero (Perkel et al., 1967; Aertsen et al., 1989; Johnson and Alloway, 1996; Roy and Alloway, 1999). To determine whether thalamocortical interactions were statistically significant, we calculated a shift predictor (Gerstein and Perkel, 1972; Johnson and Alloway, 1996). The shift predictor represents the expected value of the CCG attributable to chance and stimulus-induced coordination, and a peak in the raw CCG was considered statistically significant only if it exceeded the shift predictor by at least $1.96 \mathrm{SDs}$ (i.e., the $95 \%$ confidence limits). An SD in the shift predictor is equal to its square root (Aertsen et al., 1989), and we determined the $95 \%$ confidence limits by adding the shift predictor to the product of 1.96 times the square root of the shift predictor.

The strength of thalamocortical interactions was calculated from an efficacy ratio (Levick et al., 1972). In essence, the efficacy ratio indicates the proportion of reference events that result in subsequent time-locked discharges in the target neuron. This relationship is expressed by the equation: efficacy $=$ target events/total reference events. To measure thalamocortical efficacy, we divided the number of events in the tallest 2 msec period of the raw CCG peak (appearing $0-4 \mathrm{msec}$ after time zero) by the number of thalamic reference events at time zero, and then multiplied this ratio by 100 to obtain a percentage. The numerator in the efficacy ratio was based on spike counts in a $2 \mathrm{msec}$ period because cortical interspike intervals were rarely $<2 \mathrm{msec}$, and this ensured that no more than one cortical discharge was counted per reference event; otherwise, in cases where multiple cortical events occurred in rapid succession, a longer time interval could allow the efficacy ratio to exceed a value of 1 . Only events occurring within $0-4$ msec of time zero were considered for our efficacy measurements because, as shown by antidromic stimulation experiments, this time lag corresponds to the conduction of thalamocortical impulses in the somatosensory system of anesthetized cats (Yen et al., 1985). Furthermore, our previous work in cat SI indicated that most thalamocortical interactions in the middle cortical layers transpire in this time period, whereas thalamocortical interactions involving the extragranular layers tend to have a much larger portion of their responses that transpire over longer time lags or occur before time zero (Johnson and Alloway, 1996).

To determine the amount of coordinated activity among pairs of simultaneously recorded thalamic neurons, we also constructed raw CCGs of thalamic activity and displayed them with respect to $95 \%$ confidence limits as described above. Instead of constructing an efficacy ratio for these CCGs, we calculated the correlation coefficient, because this parameter is more appropriate for pairs of neurons that are unlikely to have serial connections. The formula for calculating the crosscorrelation coefficient, $p(\tau)$, was similar to the formula used by Eggermont (1992):

$$
p(\tau)=\frac{[C E]}{\sqrt{\left(\left[N_{\mathrm{T}}-\left(\left(N_{\mathrm{T}}\right)^{2} / T\right) \Delta\right]\left[N_{\mathrm{R}}-\left(\left(N_{\mathrm{R}}\right)^{2} / T\right) \Delta\right]\right)}},
$$

where $C E$ is the number of near-coincident events in the tallest $2 \mathrm{msec}$ period of the raw CCG peak, $\Delta$ represents the time interval for measuring $C E$ ( $2 \mathrm{msec}), T$ is the total time over which stimulus-induced discharges were recorded $(600,000 \mathrm{msec})$, and $N_{\mathrm{T}}$ and $N_{\mathrm{R}}$ represent the total number of discharges from the target and reference neurons that were recorded during time $T$. In contrast to studies in which $C E$ represents the number of excessive coincident events after subtracting a shift predictor (Eggermont, 1992), the numerator of our correlation coefficients was based on all near-coincident events, not just the portion exceeding the shift predictor. It is important to note, however, that we analyzed only those neuronal trios in which subtraction of the shift predictor had already shown that the number of near-coincident events in the thalamocortical CCGs was statistically greater than could be predicted from the underlying rate of activity (see above).

To display the relative timing of discharges among a trio of thalamic 
and cortical neurons (two thalamic neurons paired with an SII cortical neuron), we constructed snowflake histograms from the interspike intervals appearing across each pair of spike trains (Gerstein and Perkel 1972; Perkel et al., 1975). By definition, the central origin of each snowflake histogram represents instances in which all three neurons discharged simultaneously. In the present study, we constructed each snowflake histogram so that synchronous thalamic events were represented by points along the horizontal time axis. In addition, cortical events that occurred after synchronous thalamic events were always represented to the right of the central origin.

\section{RESULTS}

We recorded stimulus-induced responses of 90 thalamic and 85 SII cortical neurons in 36 experiments conducted across 13 separate recording sessions (6 sessions in the first cat and 7 sessions in the second cat). In a typical recording session, the cortical or thalamic electrodes were advanced once or twice so that multiple experiments could be conducted within each recording session. Different cortical neurons were often recorded at two or three depths within the same electrode penetration, but successive recordings within a penetration were separated by only 150-300 $\mu \mathrm{m}$.

Waveform analysis revealed that multiple isolated neurons were usually recorded from each electrode. We recorded an average of $1.74 \pm 0.129$ (mean \pm SEM) neurons at each thalamic recording site, whereas an average of $1.81 \pm 0.119$ neurons were recorded from each cortical recording site. After sorting neuronal waveforms into distinct clusters, we identified a total of 360 neuronal trios in which two thalamic neurons were recorded simultaneously with an SII neuron on three separate electrodes. The number of neuronal trios exceeded the number of experiments $(n=36)$ because multiple neurons were often recorded simultaneously from an electrode and a given neuron could be an element of more than one trio. For example, if two electrodes were placed in both cortex and thalamus, an experiment in which a pair of neurons were recorded from each of the four electrodes would yield a total of 16 neuronal trios that were recorded simultaneously. Conventional cross-correlation analysis was then used to identify all neuronal trios that showed significant levels of stimulus-induced coordination in all pairwise combinations. This meant that each neuron in the trio had to display significant levels of coordination with the other two neurons during one or more of the three stimuli (airjet 1 or airjet 2 or combined airjet stimulation), but not necessarily in response to the same stimulus. After applying these criteria to our sample of 360 neuronal trios, we identified 26 trios that showed significant levels of thalamic synchronization and thalamocortical coordination. These 26 neuronal trios involved a total of 40 thalamic and 19 cortical neurons; among these 26 trios, 18 were composed of neurons that appeared in more than one trio.

A typical example of the responses recorded from one of the neuronal trios displaying both thalamic synchronization and thalamocortical coordination is illustrated in Figure 1. As depicted in Figure $1 A$, the neurons had overlapping receptive fields on the hairy skin of the distal forelimb, and the airjets were positioned so that each stimulus evoked responses from all three neurons. As demonstrated by the conventional CCGs in Figure $1 C$, correlated discharges in the thalamus and cortex were considered significant only if they exceeded the $95 \%$ confidence limits at lag times (i.e., $0-4 \mathrm{msec}$ ) that were consistent with the conduction time of thalamocortical impulses in the feline somatosensory system (Yen et al., 1985). Thalamic synchronization, however, was always characterized by a peak of correlated events at time zero in the CCG (Alloway et al., 1995). For trios in which thalamic activity was strongly correlated, the CCG had a tall, narrow peak at time zero that was only $1 \mathrm{msec}$ wide (Fig. 2, TC15, TC16). By comparison, weakly correlated thalamic activity was often represented by small, broader CCG peaks that were 2-5 msec wide and straddled time zero (Fig. 2, TC4).

We also constructed autocorrelation histograms (ACGs) over longer time periods $( \pm 250 \mathrm{msec})$ to determine whether the pairwise interactions detected in the CCGs were caused by phaselocked neuronal oscillations. In a small fraction of cases we observed oscillations in the spontaneous activity of thalamic and cortical neurons, but we never observed such oscillations during the stimulus-induced responses. Instead, the stimulus-induced ACGs showed large peaks at time zero and, on occasion, one or two additional small peaks for the next 50-100 msec, but we never observed periodic changes in activity over the entire analysis interval ( $\pm 250 \mathrm{msec})$.

\section{Snowflake analysis}

Snowflake histograms revealed several findings about thalamic synchronization and its relationship to cortical responsiveness. As indicated by Figure 2, neuronal trios with substantial amounts of thalamic synchronization were usually characterized by a prominent band of coordinated events along the horizontal time axis of the snowflake histogram (Fig. 2, TC15, TC16). If the thalamic neurons were weakly correlated, however, the horizontal time axis was not as prominent and often had a fragmented appearance (TC12 and TC4 in Figs. 1 and 2, respectively). Despite these variations in the strength of thalamic synchronization, the probability of a cortical discharge was usually highest immediately after both thalamic neurons discharged simultaneously. This pattern of coordination was indicated by the presence of white bins on the horizontal time axis that were located to the right of the central origin. Given that thalamic synchronization is represented by the horizontal time axis, the distance to the right of the central origin indicates the time lag between synchronous thalamic discharges and subsequent cortical discharges (Fig. 1D).

The timing of cortical facilitation in the snowflake histograms was usually consistent with the timing of thalamocortical interactions. In many snowflake histograms, the horizontal time axis, which represents thalamic synchronization, was intersected by diagonal bands depicting thalamocortical interactions (Figs. 1, 2, blue arrows). These diagonal bands represent instances in which thalamocortical interactions involving one thalamic neuron occurred independent of discharges in the other thalamic neuron, and these interactions usually involved time lags of $0-4 \mathrm{msec}$ from the synchronous thalamocortical time axes (Fig. 1D, diagonal red lines). In cases where these diagonal bands were apparent in the snowflake histogram, cortical responsiveness was highest at the region where these diagonal bands intersected the horizontal time axis.

Changes in stimulus configuration produced slight variations in the pattern of thalamocortical coordination that appeared in the snowflake histograms. As indicated by Figure 3, one effect of changing stimulus location was to alter the incidence and spatial extent of responses in the snowflake histogram. When compared with the response to airjet 1 , for example, the second airjet in experiment TC15 produced smaller responses in the PSTHs and a corresponding decrease in the spatial extent of responsiveness in the snowflake histogram (Fig. 3C). Despite these stimulusinduced variations, similar patterns of thalamocortical coordination were apparent in the snowflake histograms for the individual stimuli and their summed responses (Fig. 2, middle panel). Thus, cortical responsiveness was noticeably higher immediately after 

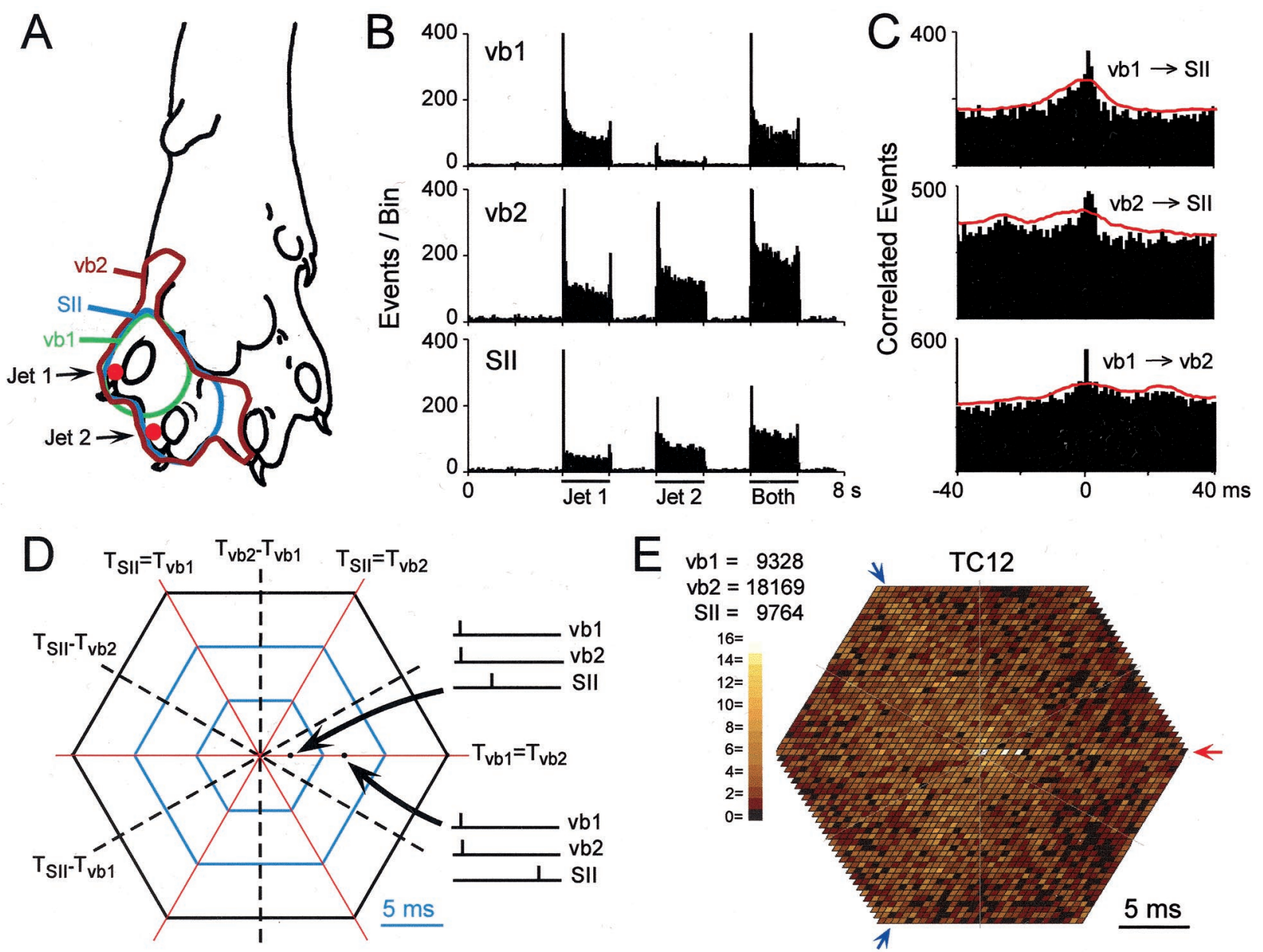

Figure 1. Representative experiment illustrating relationship between thalamic synchronization and thalamocortical coordination (experiment TC12). $A$, Receptive fields for neurons in the ventrobasal complex $(v b 1, v b 2)$ and secondary somatosensory cortex (SII). Red circles indicate airjet stimulation sites. $B$, PSTHs of neuronal responses to 200 trials of airjet stimulation. Binwidths, 25 msec. $C$, Raw CCGs displaying significant amounts of correlated activity as indicated by peaks exceeding the $95 \%$ confidence limits (red lines). Each CCG was based on the sum of discharges occurring in response to all stimulus configurations. Binwidths, 1 msec. $D$, Template indicating how patterns of thalamic and cortical activity are displayed in the snowflake histograms. Dashed lines represent time axes for displaying interspike intervals for each pair of neurons (e.g., $\left.T_{S I I}-T_{v b 1}\right)$. Red lines represent synchronous time axes for displaying instances in which neurons discharge at the same time (e.g., $T_{v b 1}=T_{v b 2}$ ); points along the horizontal red line indicate instances of thalamic synchronization. Blue lines depict concentric hexagons that represent $5 \mathrm{msec}$ intervals along each interspike interval axis. As shown by the spike trains and their corresponding points in the template, synchronized thalamic events and subsequent cortical discharges appear to the right of the central origin. E, Summed snowflake histogram for experiment TC12 based on discharges occurring in response to all stimulus configurations. White bins on the horizontal time axis (red arrow) reflect a large number of cortical discharges that occurred immediately after the thalamic neurons discharged simultaneously. Thalamocortical interactions for each thalamic neuron are indicated by faint diagonal bands (blue arrows). Total stimulusinduced discharges and a color-coded legend appear at the left. Binwidths, $0.5 \mathrm{msec}$.

synchronized thalamic discharges regardless of the stimulus configuration. The major change produced by different stimuli was a small shift in the oblique band of thalamocortical interactions from a lag of 1-2 msec for airjet 1 to a lag of 3-5 msec for airjet 2. Presumably, this shift reflects the fact that the second airjet was closer to the receptive field edge of the cortical neuron, and this caused a slight increase in response latency. The fact that different stimulus configurations produced greater variability in the relative timing of thalamic discharges was advantageous for our study because it provided us with a wider distribution of nearcoincident thalamic discharges that could be used to determine the effective interval over which the SII neurons integrated thalamocortical inputs (see below).

In contrast to the moderate changes in coordination produced by different stimulus configurations, a comparison of the summed snowflake responses obtained across different neuronal trios re- vealed considerable variability in thalamocortical coordination. As shown by a wide range of snowflake histograms in Figure 4, the summed coordination patterns could vary substantially across neuronal trios, even among those that shared some of the same neurons. Although thalamic synchronization and thalamocortical coordination exceeded the $95 \%$ confidence limits in the pairwise CCGs generated from individual stimuli, sometimes these interactions were not prominent in the summed snowflake histograms. In these cases, the interactions were either too weak or were diluted in the summed snowflake response because they occurred during only one stimulus configuration. Despite these variations, many snowflake histograms contained similar features that could be classified into a few distinct categories. As described earlier, the most common pattern of coordination was characterized by a prominent horizontal band through the origin, indicating the presence of large amounts of thalamic synchronization (e.g., 

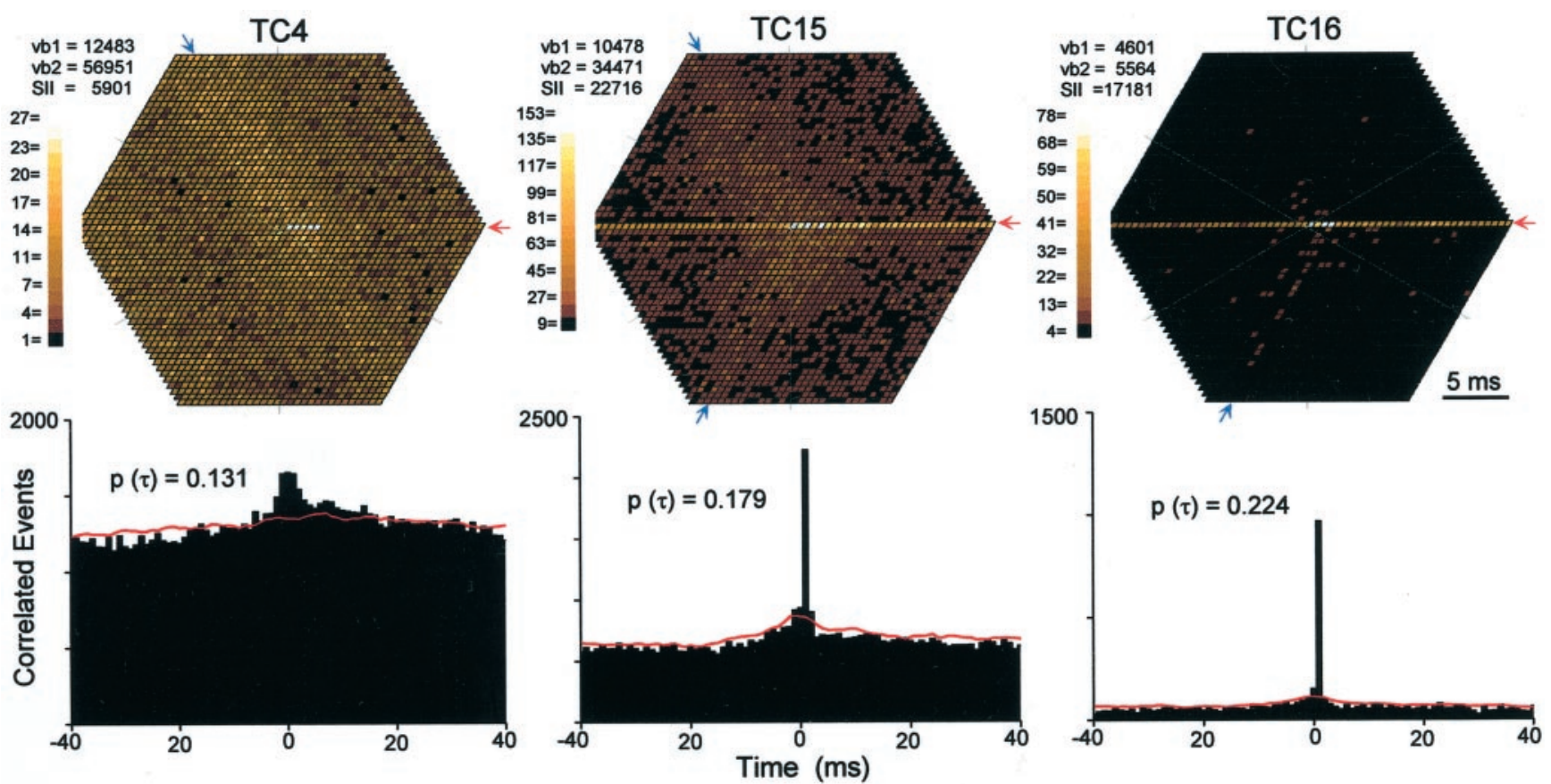

Figure 2. Cortical responses to varying amount of thalamic synchronization. Top panel, Summed snowflake histograms, illustrated as in Figure 1, displaying coordinated activity for three separate experiments (TC4, TC15, and TC16). Binwidths, 0.5 msec. Bottom panel, Raw CCGs indicating the proportion of thalamic activity that was synchronized in these experiments. The correlation coefficients appear next to the CCG peak. Binwidths, 1.0 msec.

TC17, TC19, TC44). In a smaller number of cases, a fragmented horizontal band was present (e.g., TC13 and TC14) that indicated weaker levels of thalamic synchronization. Another common feature was the presence of one or two oblique bands representing strong thalamocortical interactions (e.g., TC5a and TC14a). The least common coordination pattern was represented by a diff use cloud of enhanced responsiveness to the right of the snowflake origin (e.g., TC20). Of course, many snowflake histograms contained combinations of these different coordination patterns, and this accounted for much of the variation seen across the different neuronal trios. Despite these apparent variations in thalamocortical coordination, virtually all of the snowflake histograms were characterized by increased cortical responsiveness immediately after coincident thalamic discharges. Comparison of the summed snowflake histograms in Figures 1, 2, and 4 indicate that thalamic synchronization and subsequent cortical facilitation represent relatively isolated events that occurred much more frequently than the surrounding coordination patterns. This conclusion is based on the fact that the white bins in the snowflake histograms tend to be surrounded by bins with color-coded magnitudes at least $50 \%$ lower (i.e., brown or black bins). Only in rare instances (e.g., TC47) did we observe cases where the white bins along the thalamic synchronization axis were surrounded by a large number of coordination patterns that had similar magnitudes (i.e., yellow or orange bins).

\section{Conditional cross-correlation analysis}

Snowflake histograms are appropriate for displaying the temporal pattern of coordination among three simultaneous neurons, but they have some disadvantages that limit their usefulness for determining the impact of thalamic synchronization on cortical responsiveness. First, as indicated by Figure 5, snowflake histograms are unable to display instances in which only two of the three neurons discharge within the snowflake analysis interval ( $\pm 15 \mathrm{msec}$ in our snowflake histograms). Furthermore, a pair of points in the snowflake histogram do not necessarily represent a unique pattern of coordinated activity but could represent several different combinations of spike trains. As illustrated in Figure 5, $C$ and $D$, separate instances of heterosynaptic integration $(C)$ could produce the same pattern of points in the snowflake histogram as a combination of homosynaptic and heterosynaptic integration $(D)$. To measure the impact of thalamic synchronization on cortical responsiveness, however, it is necessary to analyze instances of heterosynaptic integration that are isolated from instances of homosynaptic integration.

To determine the impact of thalamic synchronization on cortical responsiveness we developed a computer program to perform conditional cross-correlation analysis. Initially, as shown in Figure $6 A$, our program sorted the discharges in the two thalamic spike trains into distinct categories: either both thalamic neurons discharged relatively close in time or one thalamic neuron discharged by itself. Thus, after encountering a discharge in the spike train of one thalamic neuron, the computer program examined the other thalamic spike train to determine whether it also contained a discharge within a "search interval" of a specific duration. After completing this process using one thalamic spike train as the source of reference events, this process was repeated using the other thalamic spike train as the source of reference events. As shown in Figure $6 A$, the search interval was always centered around the reference discharge encountered in one of the thalamic spike trains, and pairs of thalamic events were considered "synchronous" if they occurred within an interval equal to half the width of the search interval. These events were then sorted into four different groups (i.e., synchronous events using VB1 as the reference point, synchronous events using VB2 as the reference point, VB1 events only, or VB2 events only), and the time stamps of the reference thalamic spikes were then correlated with the time stamps of the cortical spike train to generate four separate CCGs (i.e., two synchronous CCGs and 

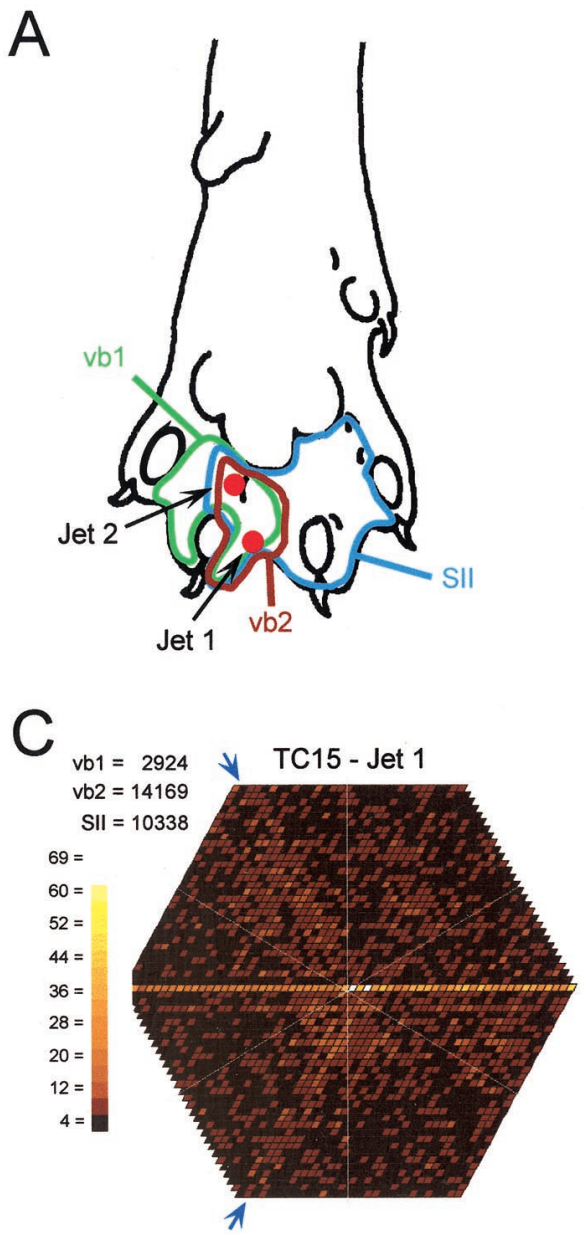
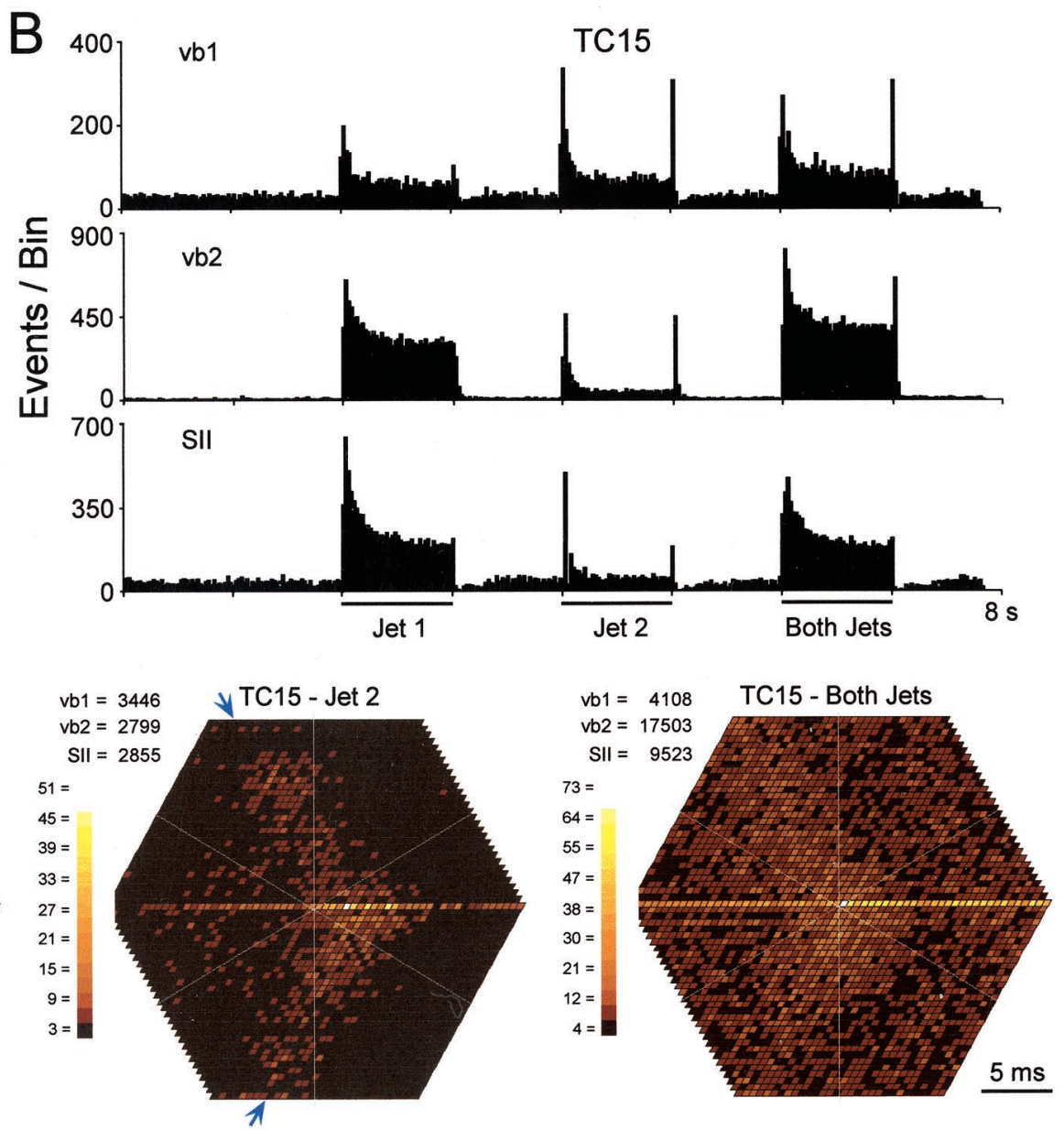

Figure 3. Effects of different stimuli and their combined administration on the coordination of thalamic and cortical activity in experiment TC15. A, Receptive fields and airjet stimulation sites. $B$, PSTHs illustrating neuronal responses in the ventrobasal thalamus and SII cortex during 200 stimulation trials. $C$, Snowflake histograms illustrating how thalamocortical coordination varied in response to different airjets or their combination. All panels illustrated as in Figure 1.

two asynchronous CCGs). Because the ventrobasal thalamus and SII cortex are serially connected (Spreafico et al., 1981; Burton and Kopf, 1984), this allowed us to calculate an efficacy ratio to indicate the probability that a thalamic reference event will be followed by a subsequent cortical event (Levick et al., 1972; Aertsen et al., 1989).

Representative results from using conditional cross-correlation analysis to analyze thalamocortical efficacy as a function of synchronous and asynchronous thalamic events are illustrated for one neuronal trio in Figure $6 \mathrm{~B}$. Because the pair of CCGs generated from the synchronous thalamic events were virtually identical, an average of the two synchronous CCGs is presented for each search interval in Figure $6 B$. It is evident from Figure $6 B$ that extremely short search intervals (i.e., $1 \mathrm{msec}$ ) resulted in the identification of fewer instances in which both thalamic neurons discharged synchronously. As the search interval was lengthened, however, an increasing number of instances were identified in which both neurons discharged synchronously (i.e., within the search interval). By comparison, the search for asynchronous thalamic discharges was most productive when the search interval was extremely short. As the search interval was lengthened, however, the number of asynchronous discharges gradually declined because there were fewer occasions in which either thalamic neuron discharged in isolation. Regardless of the length of the search interval, thalamocortical efficacy was always higher for synchronous than for asynchronous thalamic discharges. This is reflected by the fact that prominent peaks were present in all of the CCGs that were based on synchronous thalamic events but were not present in the asynchronous CCGs if the search intervals were $>16$ msec (i.e., discharges in the two thalamic neurons were separated by $>8 \mathrm{msec})$.

A comparison of the mean efficacy values obtained from all 26 neuronal trios revealed that synchronous thalamic events were substantially more effective for evoking cortical activity than asynchronous events. As Figure 7 illustrates, when the search interval was only $1.0 \mathrm{msec}$, the mean efficacy of synchronous thalamic events was almost twice as large as that produced by asynchronous events (12.6 vs $6.9 \%$ ). The efficacy of synchronous events gradually declined with longer search intervals and eventually became indistinguishable from the mean efficacy rate that was obtained by conventional crosscorrelation analysis. The fact that progressively longer search intervals produced a systematic decline in thalamocortical efficacy indicates that cortical responsiveness is influenced by the relative timing of thalamic inputs. Unfortunately, the precise interval over which cortical neurons may effectively integrate inputs from different thalamic neurons could not be determined from the method used to generate the data appearing in 

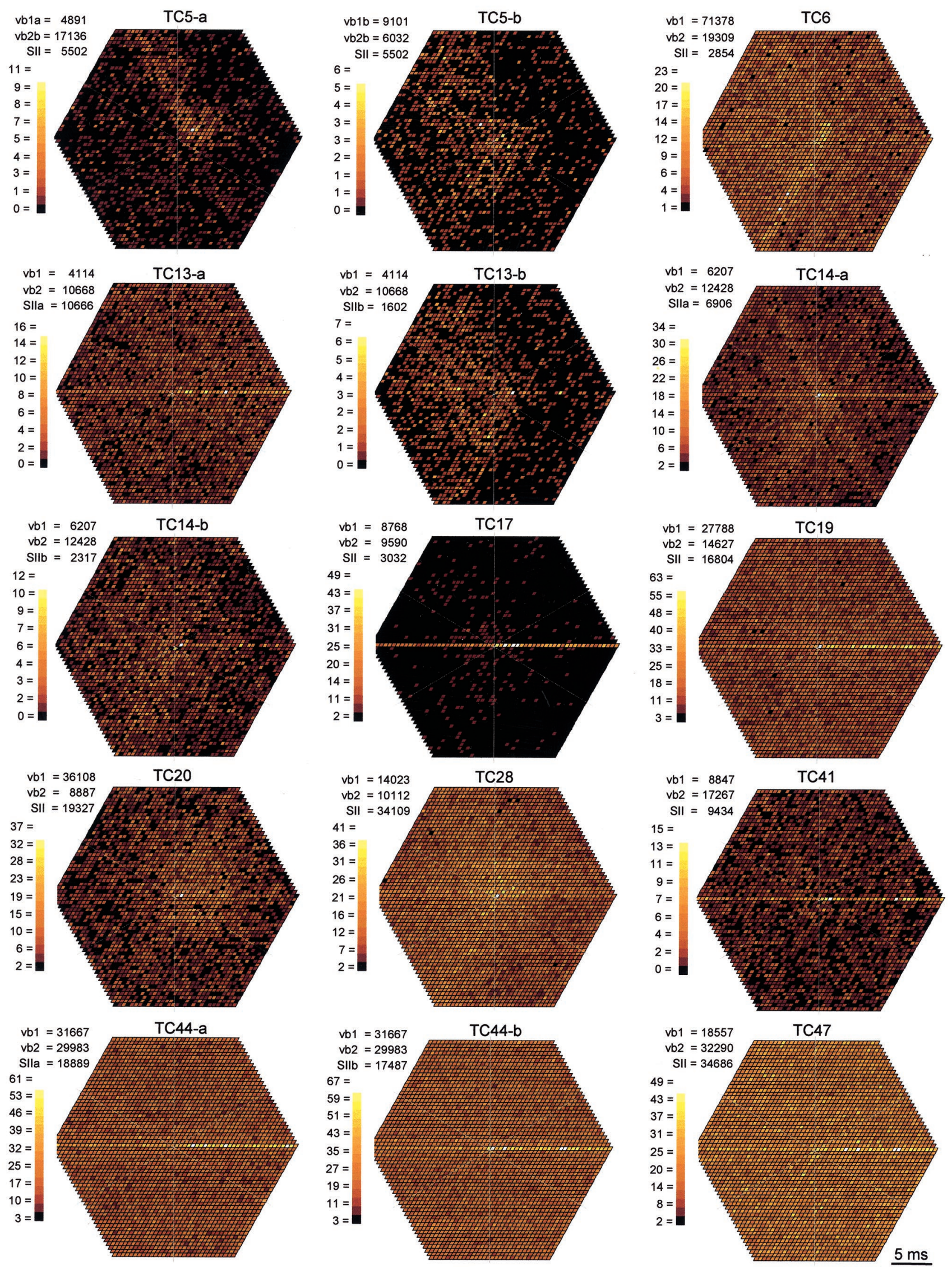

Figure 4. Variations in the pattern of thalamocortical coordination recorded across different neuronal trios. Each snowflake histogram represents the summed response to all stimulus configurations as shown in Figures 1 and 2. Some recording experiments are represented by more than one snowflake histogram because multiple neurons were often recorded from the two thalamic electrodes (e.g., TC5) or from the electrodes in SII cortex (e.g., TC13, TC14, and TC44). 

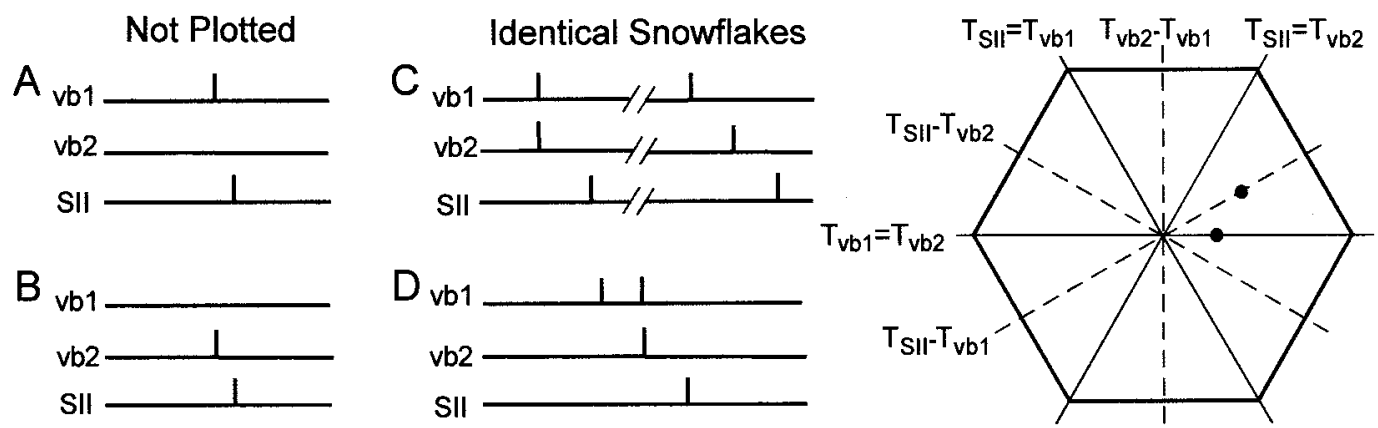

Figure 5. Limitations of snowflake histograms for depicting thalamocortical interactions. $A$ and $B$ illustrate two sets of spike trains that cannot be plotted in a snowflake histogram because only two of the three neurons discharged within the analysis interval ( $\pm 15 \mathrm{msec})$. $C$ and $D$ illustrate two sets of spike trains that are depicted by an identical pair of points in the snowflake histogram shown to the right. Hence, identical data points in a snowflake histogram may represent two isolated instances of heterosynaptic integration $(C)$ or an instance in which homosynaptic and heterosynaptic integration are combined $(D)$.

Figure 7, because long search intervals allowed considerable variation in the relative timing of the thalamic discharges. With a search interval of $64 \mathrm{msec}$, for example, thalamic discharges across the two neurons could be separated by variable amounts of time, ranging from 0 to $32 \mathrm{msec}$, and still be identified as being synchronous. Thus, our initial search algorithm only determined whether both thalamic neurons discharged within a specific interval, sometimes of relatively long durations, and did not sort cortical responses according to the precise interval between thalamic discharges.
A

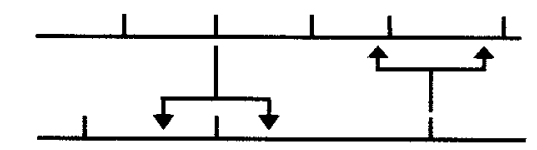

Search intervals filled
VB1

VB2

$1 \mathrm{~ms}$ Search

B
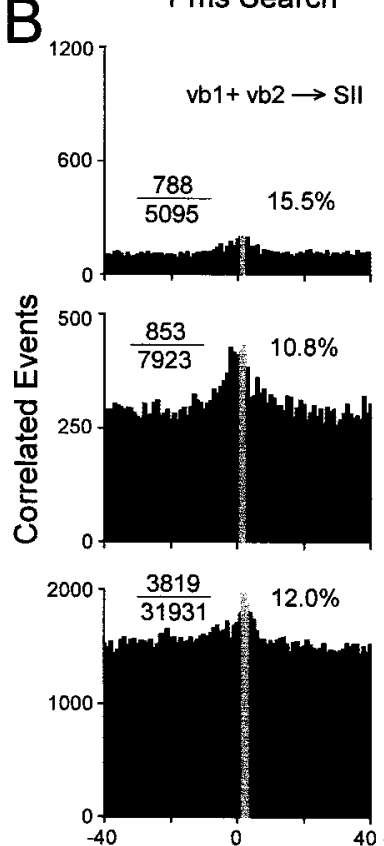

$4 \mathrm{~ms}$ Search
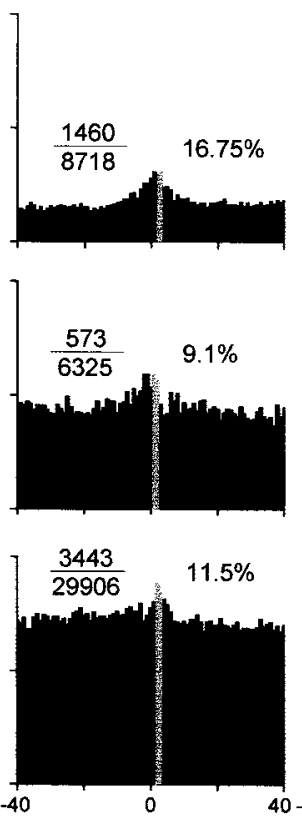

\section{Asynchronous Events}

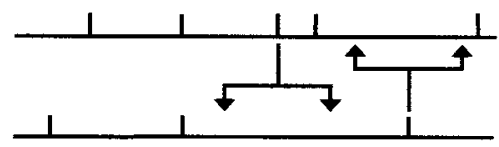

Search intervals empty
$16 \mathrm{~ms}$ Search
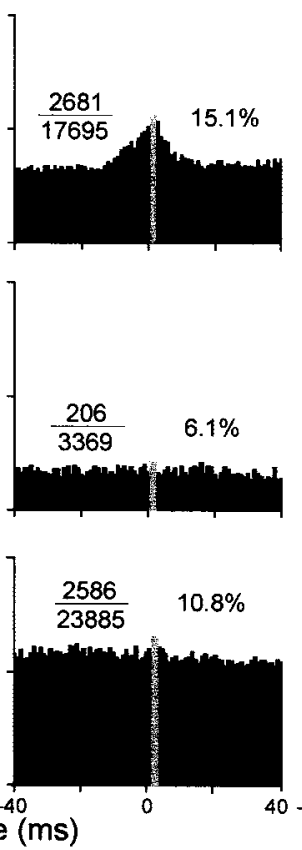

$64 \mathrm{~ms}$ Search
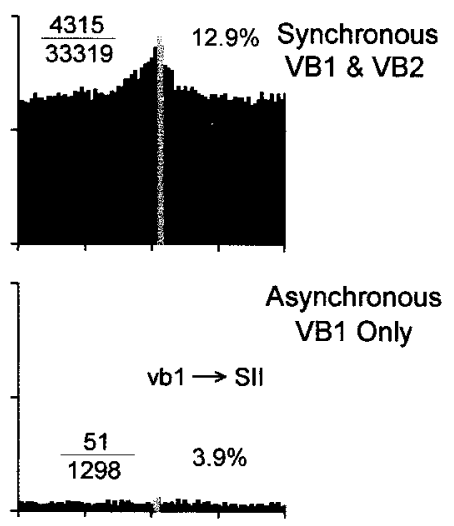

Asynchronous VB1 Only

$$
\text { (1) }
$$

(n) 


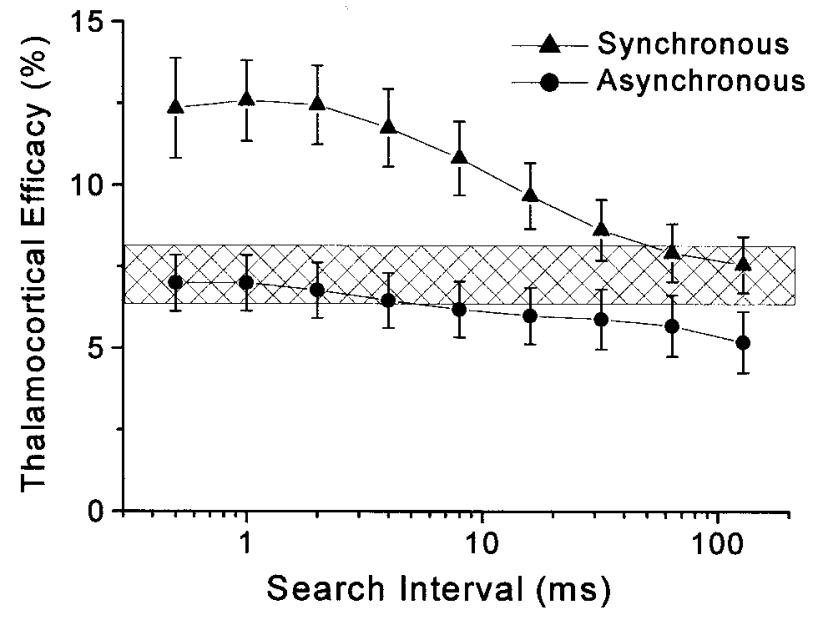

Figure 7. Changes in thalamocortical efficacy as a function of search interval duration. Each line shows the mean $( \pm$ SEM) rate of thalamocortical efficacy calculated from the conditional CCGs of 26 neuronal trios. The horizontal cross-hatched bar represents the mean ( \pm SEM) thalamocortical efficacy obtained by using conventional cross-correlation analysis.

\section{Interneuronal interspike interval analysis}

To determine more precisely the effective interval for cortical integration of different thalamic inputs, we constrained our search strategy as shown in Figure $8 A$. First, we limited the duration of the search intervals (i.e., $1-5 \mathrm{msec}$ ) to reduce the uncertainty about the relative timing of discharges across the thalamic neurons. Second, the search interval was offset backward in time from the reference discharge so that we could identify specific instances in which discharges in the two thalamic spike trains were separated by a specific interval without any intervening discharges [i.e., an interneuronal interspike interval (INISI)]. Third, an INISI search was considered successful only if both neurons failed to discharge during deadtime intervals located immediately before and after the INISI. This last constraint effectively identified instances in which cortical responses occurred after isolated heterosynaptic events (Fig. 5). The program used both thalamic spike trains as the source of reference events, and after identifying instances in which specific INISIs were isolated from other discharges by the deadtime intervals, these reference events were correlated with the cortical spike train to generate a conditional CCG.

In this analysis we searched backward in time from the reference thalamic discharge for several reasons. First, we could not make any a priori assumption about the time interval over which thalamic inputs to an SII neuron could be integrated with subsequent heterosynaptic inputs. Previous studies, however, indicated that thalamocortical impulses require only 1-4 msec to be transmitted to the middle cortical layers (Yen et al, 1985; Johnson and Alloway, 1996), and these results were consistent with our crosscorrelation analysis of the thalamocortical interactions in SII (Fig. 1). Hence, by using the second thalamic discharge as the reference event, it was appropriate to use the subsequent 4 msec time period in the conditional CCG to calculate thalamocortical efficacy. Alternatively, if the program searched forward in time from the thalamic reference event for subsequent thalamic discharges, the uncertainty about the effective integration interval would not allow us to restrict our thalamocortical efficacy measurements to a specific time frame in the conditional CCG.

The results of this analysis are illustrated for a trio of neurons in Figure $8 B$. In this example, a short search interval of $1 \mathrm{msec}$ was used to identify instances in which the thalamic neurons discharged at a range of INISIs shown above each CCG. As these CCGs indicate, the cortical neuron had a $9.2 \%$ probability of discharging when discharges in the two thalamic neurons were separated by $\leq 1 \mathrm{msec}$, but only discharged $4.0 \%$ of the time when the intervening interval was 6-7 msec long. For longer INISIs, the probability of a cortical discharge remained relatively flat and did not decline any further (data not shown). The decline in thalamocortical efficacy to an asymptotic level indicates that this cortical neuron could integrate inputs from these thalamic neurons only if both neurons discharged within a $6 \mathrm{msec}$ interval.

To determine the effective interval for heterosynaptic integration, we identified neuronal trios in which pairs of thalamic neurons displayed cooperative effects in driving cortical activity because these trios are the most likely to have direct, monosynaptic thalamocortical connections. Thalamocortical coordination was defined as being cooperative if thalamic synchronization caused the cortical neuron to discharge at a rate greater than could be predicted from the efficacy rate obtained when each thalamic neuron discharged independently. To understand this operational definition, consider thalamic neurons whose independent (or asynchronous) discharges have a $10 \%$ chance of being correlated with subsequent time-locked discharges in a postsynaptic cortical neuron. This interaction can be expressed as an efficacy of $10 \%$ or, alternatively, as a failure rate $(F)$ of $90 \%$. If both thalamic neurons have independent failure rates of $90 \%$ for the same cortical neuron, the probability that simultaneous discharges in both thalamic neurons would also fail to elicit a time-locked cortical discharge is predicted to be $(0.9)^{2}$ or $81 \%$. Therefore, the expected probability of a time-locked cortical discharge after synchronous thalamic discharges in this case would be $19 \%$ or 1 minus the joint probability of their individual failure rates $\left(1-F_{1} F_{2}\right)$. For our purposes, the independent failure rate of each thalamic neuron was equivalent to the asynchronous efficacy rate that was obtained when long search intervals (i.e., 64-128 msec) were used in the initial search strategy shown in Figure 6. Hence, pairs of thalamic neurons were considered cooperative if their synchronous efficacy rate exceeded the predicted probability (i.e., synchronous efficacy $>1-F_{1} F_{2}$ ).

Among the 26 neuronal trios that were analyzed in detail, we found 12 that displayed thalamocortical cooperativity. In these cases, we initially searched for INISIs that were surrounded by equally long deadtime intervals. As indicated by Figure $9 A$, this search paradigm indicated that mean thalamocortical efficacy was highest for synchronous thalamic discharges and gradually declined to an asymptotic level when the INISIs were $\geq 10 \mathrm{msec}$. In response to synchronous thalamic events, the mean probability of a cortical discharge in these 12 neuronal trios was $11.7 \%$ or nearly three times the efficacy level that was measured when INISIs were $\geq 10$ msec.

These results suggest that the effective duration of heterosynaptic integration extends up to $10 \mathrm{msec}$, but it is possible that homosynaptic integration may have contributed to the level of thalamocortical efficacy present in this time period. For example, in cases where the INISIs were only $2 \mathrm{msec}$, either thalamic spike train could contain additional discharges that were just outside the deadtime intervals (also $2 \mathrm{msec}$ ) but within the effective integration interval of the cortical neuron. Furthermore, by setting deadtime intervals equal to the INISIs, the analysis of long INISIs was restricted to periods of low firing rates, whereas the analysis of brief INISIs included periods of both high and low firing rates. 
A

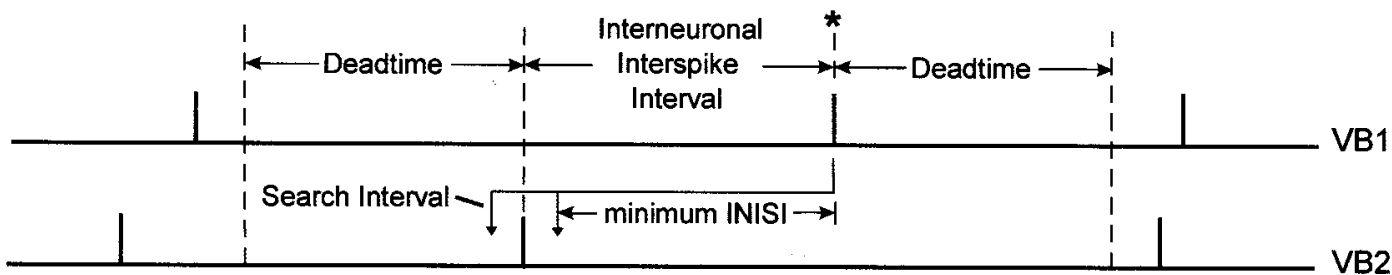

B

0-1ms

1-2ms

2-3ms

4-5ms

6-7ms
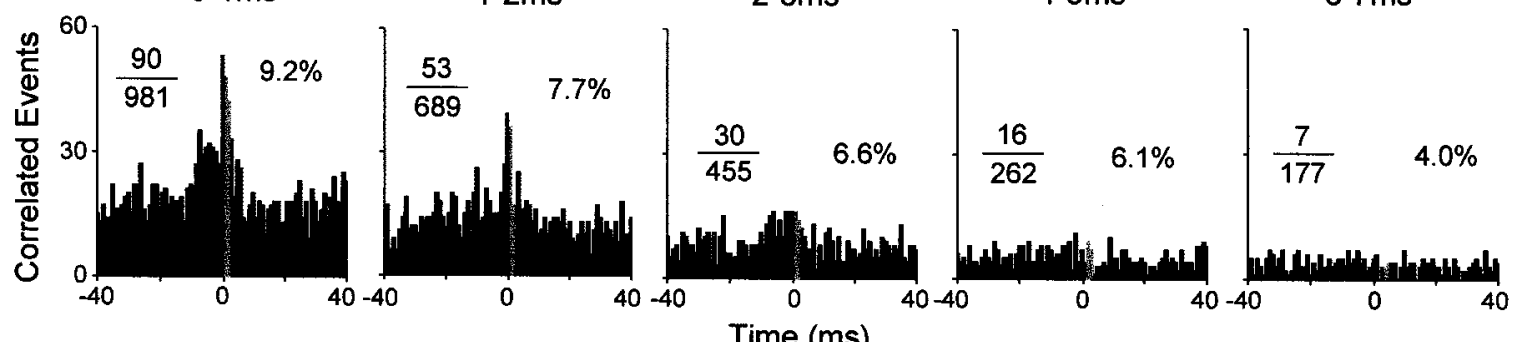

Figure 8. Thalamocortical efficacy as function of interneuronal interspike intervals (INISIs). A, Diagram illustrating the procedure for identifying instances in which discharges across the two thalamic neurons are separated by specific interspike intervals. Each spike train was used as a source of reference events, and for each discharge a search was conducted backward in time to determine whether the other thalamic spike train contained a discharge within the search interval. The discharge marked by the asterisk was used as a reference event at time zero for the conditional CCG because the other spike train contained a discharge in the search interval, and no discharges were present in either of the two deadtime intervals. $B$, Conditional CCGs constructed from identifying specific INISIs in the thalamic spike trains of experiment TC12. A search interval of 1 msec was used to identify specific ranges of INISIs as shown above each CCG; deadtime intervals were equal to the minimum INISI for each CCG. Thalamocortical efficacy appears as a ratio on the left and as a percentage on the right of each CCG. Gray regions indicate the 2 msec period used to calculate the efficacy ratio. Note that the tallest bin in some of the CCGs occurred just before time zero because the reference discharge was always the second event in a pair of thalamic discharges. Binwidths, $1.0 \mathrm{msec}$.

To remove potential instances of homosynaptic integration and reduce the bias in firing rates, we repeated the INISI searching paradigm, but required all deadtime intervals to be at least 10 msec in duration. We found that 11 neuronal trios still displayed thalamocortical cooperativity when deadtime intervals were $\geq 10$ msec, and these results are shown in Figure 9B. A comparison of Figure $9, A$ and $B$, indicates that longer deadtime intervals reduced the efficacy of near-coincident discharges. Presumably, this loss of efficacy reflects the removal of homosynaptic effects on cortical responsiveness.

After these homosynaptic influences were removed, mean thalamocortical efficacy was highest when thalamic discharges were separated by 1-2 msec. Inspection of the time course of cooperativity in each neuronal trio revealed that five trios showed the largest efficacy rates when the thalamic discharges were sep- arated by $0-1 \mathrm{msec}$, five trios were most cooperative when thalamic discharges were separated by 1-2 msec, and one trio showed the best response when the thalamic discharges were separated by $2-3 \mathrm{msec}$. The reason that some trios were most cooperative when their thalamic discharges were separated by $1-3 \mathrm{msec}$ is not clear, but these data suggest that the relative timing of somatic discharges does not represent the relative timing of synaptic inputs. Because of slight differences in the conduction velocity or length of the thalamocortical axons, it is conceivable that somatic discharges must be slightly offset in time to produce optimal synaptic inputs that have the greatest impact on cortical responsiveness.

\section{DISCUSSION}

This study revealed several major findings regarding thalamocortical communication in the somatosensory system. First, synchro-

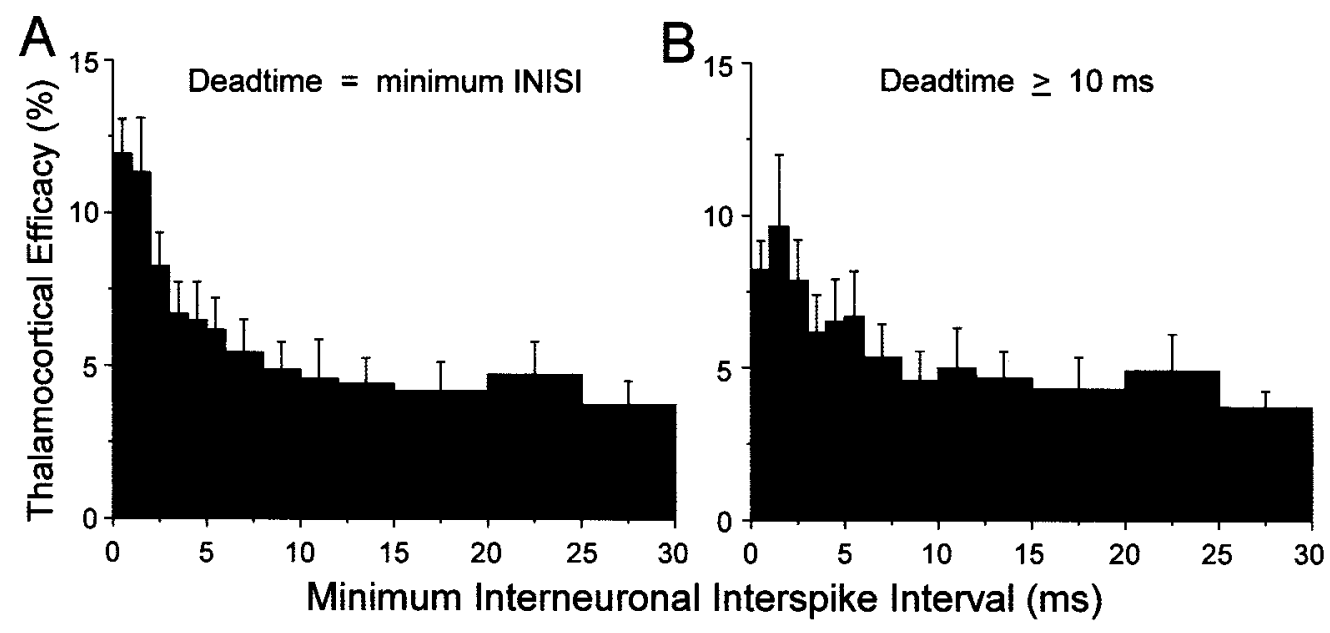

Figure 9. Thalamocortical efficacy as a function of INISI duration. $A$, Mean thalamocortical efficacy (error bars indicate SEM) calculated from instances in which deadtime intervals were equal to the minimum INISI. $B$, Same as $A$ except that deadtime intervals were $10 \mathrm{msec}$ long when the INISIs were $<10 \mathrm{msec}$. $A$ is based on 12 neuronal trios showing thalamic cooperativity; $B$ is based on 11 neuronal trios showing thalamic cooperativity. In both panels, search intervals were $1 \mathrm{msec}$ long for INISIs ranging from 0 to 6 msec but were gradually increased to $5 \mathrm{msec}$ for the longest INISIs (15-30 $\mathrm{msec}$ ) to increase the number of reference events available for conditional cross-correlation analysis. 
nous thalamic discharges are much more likely to activate somatosensory cortical neurons than asynchronous discharges. Second, thalamic cooperativity is a time-dependent phenomenon that requires near-coincident thalamic discharges to be within 6-8 msec of each other. These results support the view that neuronal synchronization may play a critical role in the transmission of sensory information from one brain region to another. This finding is especially important because any discussion about the functional value of neuronal synchronization invariably raises the issue of which neurons read the synchronization signal (Shadlen and Movshon, 1999). Our results suggest that postsynaptic neurons that receive convergent projections from the elements of a synchronized population are highly likely to be involved in computing the appropriate response to this signal.

\section{Comparisons with other experimental approaches}

Previous work examining the impact of synchronization on cortical responsiveness has relied almost exclusively on methods that simulate presynaptic synchronization. In contrast to the present study, which monitored thalamic activity in vivo, other studies either have simulated input synchrony while monitoring the discharge behavior of real cortical neurons or have used computer models to simulate both the inputs and outputs of a hypothetical neuron (Bernander et al., 1991; Stevens and Zador, 1998; Harsch and Robinson, 2000; Salinas and Sejnowski, 2000). A critical aim in these previous studies was to determine what factors mediate the irregular discharge patterns seen in most cortical spike trains (Softky and Koch, 1993). Although some results indicate that cortical discharge patterns are influenced by the parameters of neural integration (Shadlen and Newsome, 1994, 1998; Reich et al., 1997), most studies indicate that variations in the timing of synchronized inputs have a predominant effect on the temporal structure of cortical output (Bernander, et al., 1991; Stevens and Zador, 1998; Harsch and Robinson, 2000; Salinas and Sejnowski, 2000). To the extent that the timing of a cortical discharge is strongly related to its discharge probability, our results support the contention that presynaptic synchronization is a major factor in the temporal variability of cortical discharges.

Our findings corroborate results that have been observed in the visual system (Alonso et al., 1996; Usrey et al., 2000). Heterosynaptic inputs from the lateral geniculate nucleus increased the probability of activating postsynaptic targets in the striate cortex, but only when the neurons discharged within $7 \mathrm{msec}$ of each other. These data are comparable to our observations in the somatosensory system, although the thalamocortical interactions that we analyzed were, on average, much weaker than those studied in the visual system. Although we required that pairwise thalamocortical interactions exceed the shift predictor by 1.96 SDs, the results in the visual system were based on thalamocortical interactions that exceeded the expectation density by at least 6 SDs (Usrey et al., 2000). Furthermore, although thalamocortical interactions in the visual system were evoked by a drifting sinewave grating to stimulate the entire receptive field of all neurons in each trio, we used a pair of discrete airjets, both individually and in combination, to evoke thalamocortical responses. With our experimental paradigm, we found that a shift in stimulation site caused noticeable changes in the coordination of thalamic and cortical activity. Thus, by presenting each airjet stimulus both individually and in combination, we effectively increased the variability of the interspike intervals among the thalamic neurons so that we could determine the relative impact of coincident and near-coincident thalamic discharges on cortical responsiveness.
Although the experimental approaches in these two sensory systems were not identical, the results indicate that both visual and somatosensory cortical neurons are extremely sensitive to the relative timing of thalamic inputs. Furthermore, the effective interval for integrating heterosynaptic thalamocortical inputs appears to be similar in both cortical areas.

\section{Thalamocortical cooperativity}

It is widely thought that cortical spike trains depend on incoming activity from a large number of presynaptic neurons (White, 1989; Mason et al., 1991). If a population of presynaptic neurons must be active to evoke discharges from a cortical neuron, it may seem paradoxical that synchronous discharges in only two thalamic neurons could produce reliable changes in the discharge probability of a cortical neuron. Several other factors, however, must be considered. For one, the effects of synchronized thalamocortical inputs during sensory stimulation are probably superimposed on depolarization of the membrane potential of the cortical neuron. Intracellular recordings of cortical neurons have shown that sensory stimulation increases the probability that the membrane will depolarize to threshold levels (Douglas et al., 1991; Anderson et al., 2000; Carandini and Ferster, 2000), and under these conditions, computational models have shown that weakly correlated inputs can increase the discharge probability of a hypothetical neuron (Salinas and Sejnowski, 2000). This is consistent with our finding that different pairs of thalamic neurons can influence the magnitude of cortical responsiveness, although they differ substantially in the strength of their correlated activity (Fig. 2). An additional factor concerns the relative timing of discharges among the surrounding population of thalamic neurons. We have shown previously that cutaneous stimulation evokes synchronization among neighboring thalamic neurons with discharges recorded from the same electrode (Alloway et al., 1995). This strongly suggests that each of our recorded thalamic neurons was also synchronized with neighboring neurons with spike trains that were not recorded. Hence, the increase in cortical discharge probability observed here probably reflects synchronization among a local population of thalamocortical neurons.

Neuronal oscillations have been used to explain long-range synchronization between cortical areas that are not directly interconnected, but several facts argue against the possibility that the results in the present study were caused by widespread thalamic oscillations or other population dynamics that could influence cortical responsiveness independent of specific neuronal connections. First, cross-correlation analysis of pairs of thalamic neurons failed to reveal regular peaks of correlated activity that are indicative of rhythmic responses. Instead, we routinely observed narrow peaks of correlated discharges in the CCGs that were 1-4 msec in duration. Second, increased cortical responsiveness appeared in a narrow temporal interval, usually $0-4 \mathrm{msec}$ after thalamic activity, and this time lag is consistent with the conduction velocity of thalamocortical impulses. Third, thalamic synchronization and thalamocortical interactions were present only in pairs of neurons that had overlapping receptive fields. If widespread neuronal oscillations of a similar frequency were present in both thalamus and cortex, then neurons with nonoverlapping receptive fields should have displayed significant levels of correlated activity. Finally, autocorrelation analysis over long time intervals $( \pm 250 \mathrm{msec})$ failed to reveal stimulus-induced oscillations in the thalamus or cortex. These data are consistent with other findings from our laboratory showing that stimulusinduced coordination of neurons in the ventrobasal thalamus and 
somatosensory cortex does not depend on the presence of neuronal oscillations (Johnson and Alloway, 1996; Roy et al., 2001).

Although certain facts suggest that some thalamic and cortical neurons that we analyzed were probably not directly interconnected, this possibility does not alter the interpretation of our data. Thus, some of our thalamic neurons were probably interneurons or else projected exclusively to SI cortex. This situation would not alter our conclusions, however, because these types of thalamic neurons are probably synchronized with the thalamocortical neurons that do project to SII cortex (Alloway et al., 1995). It is also possible that some of our cortical neurons were located outside cortical layers IIIB or IV and did not receive a dense supply of monosynaptic thalamocortical inputs near their soma. We could not rule out this possibility because we did not make fiduciary marks at our recording sites, and consequently we were unable to reconstruct our SII recording penetrations with respect to the different cortical layers. For this reason, we restricted our analysis to trios with thalamocortical interactions that transpired within $0-4 \mathrm{msec}$ because this represents the time required for conducting thalamocortical impulses to the middle cortical layers. Serial thalamocortical interactions that involve multiple intervening synapses tend to be dispersed over wider temporal intervals, and their CCGs display broader peaks than the CCGs obtained from neurons that are monosynaptically connected (Abeles, 1991; Johnson and Alloway, 1996). Because of this temporal dispersion, thalamic neurons located two or more synapses away from their common postsynaptic targets should show cooperative effects over broader INISIs. Hence, if it was possible to selectively identify neuronal trios with thalamocortical interactions that were caused primarily by multisynaptic connections, their removal from our sample should indicate that the effective interval for integrating thalamocortical inputs is $<6-8 \mathrm{msec}$.

Finally, our data indicate that SI cortical neurons are sensitive to the relative timing of presynaptic inputs, but the effective integration interval (i.e., 6-8 msec) determined by our analysis might be specific to thalamocortical inputs and may not reflect the time course for integrating corticocortical inputs. Thalamocortical synapses are concentrated in specific cortical layers and thus are more spatially focused than corticocortical connections. This anatomical pattern may serve to enhance the effects of thalamic synchronization on cortical responsiveness, especially during high rates of thalamic activity. This possibility is suggested by the fact that the large transient responses at stimulus onset and offset tend to be transmitted more effectively to the cortex than the steadystate responses that occur during the stimulus plateau. Thus, a comparison of the PSTH responses of thalamic and cortical neurons often indicates that the ratio of the onset and plateau responses is larger for cortical than for thalamic neurons (Fig. 1, 3). Therefore, one important effect of thalamic synchronization might be an improvement in the transmission of intense or highly salient sensory signals that are coded both by high firing rates and an increased probability of coincident discharges.

\section{REFERENCES}

Abeles M (1982) Local cortical circuits: an electrophysiological study. Berlin: Springer.

Abeles M (1991) Corticonic: neural circuits of the cerebral cortex. New York: Cambridge UP.

Aertsen AMHJ, Gerstein GL, Habib MK, Palm G (1989) Dynamics of neuronal firing correlation: modulation of "effective connectivity." J Neurophysiol 61:900-917.

Alloway KD, Burton H (1985) Homotopical ipsilateral cortical projections between somatosensory areas I and II in the cat. Neuroscience 14:15-35. Alloway KD, Johnson MJ, Aaron GB (1995) A comparative analysis of coordinated neuronal activity in the thalamic ventrobasal complex of rats and cats. Brain Res 691:46-56.

Alonso JM, Usrey WM, Reid RC (1996) Precisely correlated firing in cells of the lateral geniculate nucleus. Nature 383:815-819.

Anderson J, Lampl I, Reichova I, Carandini M, Ferster D (2000) Stimulus dependence of two-state fluctuations of membrane potential in cat visual cortex. Nat Neurosci 3:617-621.

Bernander O, Douglas RJ, Martin KA, Koch C (1991) Synaptic background activity influences spatiotemporal integration in single pyramidal cells. Proc Natl Acad Sci USA 88:11569-11573.

Burton H, Kopf EM (1984) Connections between the thalamus and the somatosensory areas of the anterior ectosylvian gyrus in the cat. J Comp Neurol 224:173-205.

Carandini M, Ferster D (2000) Membrane potential and firing rate in cat primary visual cortex. J Neurosci 20:470-484.

Douglas RJ, Martin KAC, Whitteridge D (1991) An intracellular analysis of the visual responses of neurones in cat visual cortex. J Physiol (Lond) 440:659-696.

Eggermont JJ (1992) Neural interaction in cat promary auditory cortex. Dependence on recording depth, electrode separation, and age. J Neurophysiol 68:1216-1228.

Gerstein GL, Perkel DH (1972) Mutual temporal relationships among neuronal spike trains: statistical techniques for display and analysis. Biophys J 12:453-473.

Gray CM (1999) The temporal correlation hypothesis of visual feature integration: still alive and well. Neuron 24:31-47.

Harsch A, Robinson HPC (2000) Postsynaptic variability of firing in rat cortical neurons: the roles of input synchronization and synaptic NMDA receptor conductance. J Neurosci 20:6181-6192.

Johnson MJ, Alloway KD (1994) Sensory modulation of synchronous thalamocortical interactions in the somatosensory system of the cat. Exp Brain Res 102:181-197.

Johnson MJ, Alloway KD (1996) Cross-correlation analysis reveals laminar differences in thalamocortical interactions in the somatosensory system. J Neurophysiol 75:1444-1457.

Konig P, Engel AK, Singer W (1996) Integrator or coincidence detector? The role of the cortical neuron revisited. Trends Neurosci 19:130-137.

Levick WR, Cleland BG, Dubin MW (1972) Lateral geniculate neurons of cat: retinal inputs and physiology. Invest Ophthalmol 11:302-311.

Mason A, Nicoll A, Stratford K (1991) Synaptic transmission between individual pyramidal neurons of the rat visual cortex in vitro. J Neurosci 11:72-84.

Perkel DH, Gerstein GL, Moore GP (1967) Neuronal spike trains and stochastic point processes. II. Simultaneous spike trains. Biophys J 7:419-440.

Perkel DH, Gerstein GL, Smith MS, Tatton G (1975) Nerve impulse patterns: a quantitative display technique for three neurons. Brain Res 100:271-296.

Reich DS, Victor JD, Knight BW, Ozaki T, Kaplan E (1997) Response variability and timing precision of neuronal spike trains in vivo. J Neurophysiol 77:2836-2841.

Roy SA, Alloway KD (1999) Synchronization of local neural networks in the somatosensory cortex: a comparison of stationary and moving stimuli. J Neurophysiol 81:999-1013.

Roy SA, Dear SP, Alloway KD (2001) Long-range cortical synchronization without concomitant oscillations in the somatosensory system. J Neurosci 21:1795-1808.

Salinas EF, Sejnowski TJ (2000) Impact of correlated synaptic input on output firing rate and variability in simple neuronal models. J Neurosci 20:6193-6209.

Shadlen MN, Movshon JA (1999) Synchrony unbound: a critical evaluation of the temporal binding hypothesis. Neuron 24:67-77.

Shadlen MN, Newsome WT (1994) Noise, neural codes and cortical organization. Curr Opin Neurobiol 4:569-579.

Shadlen MN, Newsome WT (1998) The variable discharge of cortical neurons: implications for connectivity, computation and information coding. J Neurosci 18:3870-3896.

Softky WR, Koch C (1993) The highly irregular firing of cortical cells is inconsistent with temporal integration of random EPSPs. J Neurosci 13:334-350.

Spreafico R, Hayes NL, Rustioni A (1981) Thalamic projections to the primary and secondary somatosensory cortices in cat: single and double retrograde tracer studies. J Comp Neurol 203:67-90.

Stevens CF, Zador AM (1998) Input synchrony and the irregular firing of cortical neurons. Nat Neurosci 1:210-221.

Troyer TW, Miller KD (1997) Physiological gain leads to high ISI variability in a simple model of a cortical regular spiking cell. Neural Comput 9:971-983.

Usrey WM, Alonso J-M, Reid RC (2000) Synaptic interaction between thalamic inputs to simple cells in cat visual cortex. J Neurosci 20:5461-5467.

White E (1989) Cortical circuits. Boston: Birkhauser.

Yen CT, Conley M, Jones EG (1985) Morphological and functional types of neurons in cat ventral posterior thalamic nucleus. J Neurosci 5:1316-1338. 